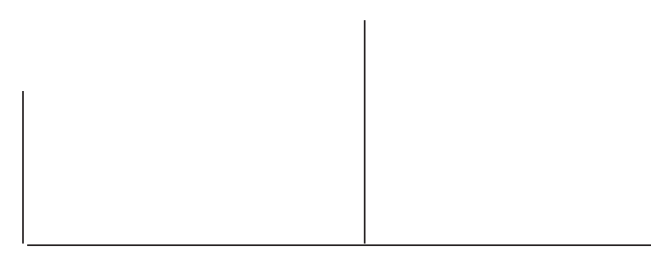

Rev. Latinoam. Psicopat. Fund., X, 3, 410-421

\title{
El concepto de self como articulador de los discursos sobre el cuerpo y la enfermedad
}

Derek Humphreys

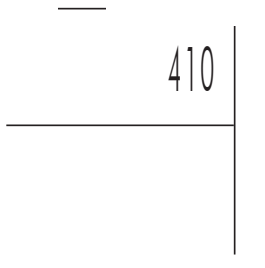

La cientificidad de la medicina moderna, al alejarse de la particularidad del caso clínico, encuentra en el concepto de Self una metáfora de individualidad. A partir de las dificultades vinculadas a este concepto, el presente artículo analiza la individualidad biológica y su relación al psicoanálisis con la intención establecer un diálogo en torno al trastorno somático. La apertura de un espacio de subjetividad, específicamente en el desafío al saber médico de los trastornos de la inmunidad, permite la introducción de una dimensión humana al sufrimiento orgánico, haciendo posible este diálogo, a través del self.

Palavras-chave: Individualidad biológica, psicoanálisis, trastorno somático, psicopatología fundamental 


\section{Introducción}

En el interés de hacer más eficaz su intervención sobre el cuerpo, a partir de mediados del siglo XIX la medicina adquiere una posición científica que la aleja paulatinamente de la particularidad de la clínica y de la experiencia subjetiva de enfermedad. Esta cientifización coincide con el surgimiento de una nueva disciplina médica, la inmunología.

A través del establecimiento de una base físico-química de individualidad, la inmunología ha dotado al modelo científico de un mito de individuación en el que cada sujeto de la especie aparece como diferente de su semejante. Esta idea de individuo no considera el aspecto subjetivo, de significación, de palabra. La aproximación a la particularidad del caso clínico de esta "materialidad" de la individuación ha tenido como efecto una recuperación, por parte de la medicina, de muchas enfermedades dichas "psicosomáticas". Sin embargo, la construcción teórica y nosológica que posibilita esta aproximación es tributaria de una cierta ambigüedad respecto de los fundamentos de la inmunidad que mantiene al paciente en una incertidumbre respecto de su enfermedad, sin permitir el establecimiento de un vínculo directo a los aspectos psicodinámicos eventualmente involucrados en ella.

Aunque el psicoanálisis, saber contemporáneo a la inmunologia, se interesa también por las dificultades de apropiación del cuerpo, de constitución de un YO diferente de lo otro y por el problema de la individuación - preguntas que delimitan la cuestión psicosomatica - éste no ha establecido las condiciones de diálogo con la medicina en torno a este problema.

A través del analisis de una experiencia clínica con pacientes afectados por una enfermedad auto-inmune, el presente articulo pretende hacer un análisis crítico del concepto biológico individualidad y sus vínculos a la subjetividad en causa en todo sufrimiento orgánico con la intención establecer un lugar para éste último al interior del hospital, en el tratamiento de los trastornos de la inmunidad. 


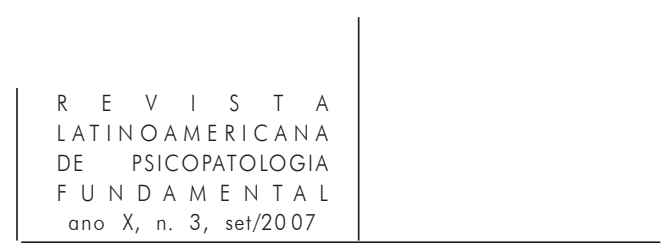

\section{La integración y la individuación como pre-textos de defensa.}

Asociada inicialmente a la infección y la investigación biológica, la inmunología es incorporada al discurso médico sólo a fines del siglo XIX, momento en el que la medicina está en plena búsqueda de un lenguaje que se adecue al modelo experimental propuesto por Claude Bernard, precisamente debido a su proximidad respecto del discurso científico. Reforzada a partir de entonces en su aspecto más concreto, la inmunología se concentrará en el establecimiento de los procesos y reacciones inmunitarios en detrimento de toda pregunta por el sentido y la finalidad - de la función primordial de la inmunidad, en tanto defensa o preservación de la individualidad - durante casi cien años.

Una vez organizada como sistema funcional, la inmunología se sitúa a la vanguardia del discurso médico durante todo el siglo XX. Primeramente, porque el sistema inmune, que no organiza su función en un órgano sino que constituye un conglomerado celular repartido en todo el organismo, sobrepasa el modelo de continuidad órgano-función-enfermedad proponiendo un nuevo paradigma fisiopatológico basado en un sistema de comunicación. Este modelo de un sistema funcional de comunicación presente de manera difusa en todo el organismo devuelve la unidad a un cuerpo dividido por los saberes parciales que resultan de la especialización médica - explosión y fragmentación en jergas especificas -, haciendo confluír los multiples saberes parciales, microscópicos, que constituyen el cuerpo médico moderno.

Esta paradigma inmunológico de un cuerpo integrado en un organismo único, que supone la existencia de una individualidad material determinable, crea la fantasía de la individuación biológica en un tiempo en el que la medicina experimental exije la exclusión de toda subjetividad de la mirada médica.

La fascinación por la investigación y descripción de este complejo conglomerado celular, lenguaje médico por excelencia del siglo XX, seguirá varias corrientes de investigación: ligada a la genética, a través de las moléculas de histocompatibilidad, incorporando el discurso cibernético en el reconocimiento de códigos moléculares individuales o asociándose a la biología molecular. En todas ellas, la inmunología ha sido capaz de adecuar perfectamente la clínica a los avances de la biología, estableciendo un diálogo perfecto con la medicina. Sin embargo, esta adecuación, que ha mantenido vigente la ilusión de la existencia de una función biológica de comunicación e integración de cada organismo individual, es consecuancia de la distancia entre el clínico y el investigador que exije la complejidad del sistema. En efecto, el clínico acepta a menudo las complejas explicaciones del investigador, que a su vez es siempre capaz de hacer una abstracción del caso, adecuándolo a un modelo general. 


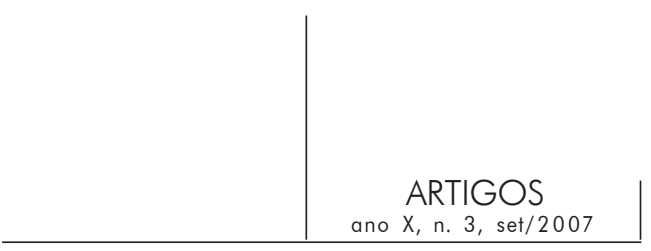

En realidad, desde la década de los noventa, consecuencia de la enorme explosión en el conocimiento en inmunología que produjo la crisis del SIDA, se ha hecho evidente para el investigador que las respuestas que este modelo otorga son más satisfactorias en términos teóricos que clínicos, con lo que algunas preguntas fundamentales respecto de la inmunidad han sido abiertas, que incluyen la cuestión de la existencia de un núcleo de individualidad - self - o de una función primordial de defensa. A pesar de este cambio radical, los fenómenos de auto-inmunidad siguen siendo, para el clínico, el efecto de un reconocimiento de constituyentes "propios" por parte del sistema inmune. Esta lógica determina, por lo demás, importantes criterios gnosológicos y terapéuticos.

\section{Self, o no self}

La inmunología, que surge a fines del siglo XIX, propone que todo viviente responde a la desarmonía intrínseca a la vida desarrollando una función de preservación de la individualidad, siendo la protección contra lo extraño secundaria a esta necesidad de homeostasis (Moulin, 1991, p. 52). Sin embargo, el interés por un reduccionismo capaz de explicar los fenómenos biológicos en términos físico-químicos hizo prevalecer la función defensiva durante todo el sigo XX. Es en este contexto que la teoría del horror autotoxicus, que establece que una ley natural de auto conservación evita que el organismo actúe contra los elementos que lo constituyen, es propuesta en 1906 (ibid., p. 60). Esta solución dogmática y simple permitió que la investigación se concentrara en la descripción de los procesos inmunitarios, dejando de lado la pregunta por la causalidad durante la primera mitad del siglo XX.

Cuando el problema de la individualidad resurge con las primeras experiencias de transplantes y los problemas de anafilaxis, Frank MacFarland Burnet propuso, en la década de los 40, una hipótesis que marcará definitivamente la inmunología: todo individuo posee un repertorio antigénico característico, el self, que el sistema inmune aprende a tolerar. El sistema inmune reconoce y destruye toda molécula extraña o no-self (Burnet, 1959). Aunque esta simplificación fue suficiente para continuar con la investigación, la hipótesis de Burnet presenta dos grandes problemas. Primeramente porque, con la intención de eliminar una discusión biológica respecto de la individualidad, Burnet introduce un término filosófico, que tiene sus propias dificultades y contradicciones, al discurso científico. Por otra parte esta hipótesis exije demasiadas excepciones para constituir una ley - basta pensar en la producción de auto-anticuerpos, fenómeno corriente que resulta inpensable en el modelo de Burnet. 


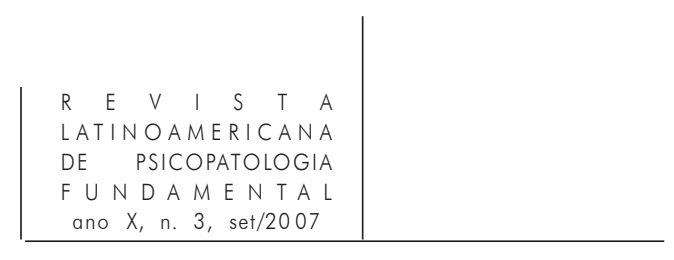

Diversas corrientes de pensamiento han intentado, a lo largo del siglo XX y hasta hoy, establecer una teoría capaz de integrar la totalidad de los fenónmenos inmunitarios. Sin poder extenderenos sobre este complejo problema, discutido en publicaciones precedentes (David-Ménard \& Humphreys, 2006), nos parece importante al menos señalar que durante los últimos 10 años el discurso científico ha intentado deshacerse del paradigma del self y de sus implicancias filosóficas a través de la teoría del "peligro" (Matzinger, 1994), que no hace más que resurgir el antiguo modelo de la inflamación sin aportar una solución adecuada.

A pesar de la ausencia de respuesta a este respecto, el concepto de self no sólo sigue siendo largamente aceptado sino que sirve hoy de articulador a la cuestión de la individualidad en tanto metáfora de una esencia trascendental identitaria estable. Pero esta fantasía identitaria es sólo posible en la nebulosa epistemológica y teórica que rodea el concepto de self. En efecto, definir el self “... desde un punto de vista exterior, como la totalidad de las moléculas constituyentes del organismo y que será desconocido por el sistema inmune..." (Tauber, 1994, p. 173), es científicamente inaceptable.

Aunque los investigadores en ciencias biológicas han intentado integrar todas las observaciones que conciernen la reacción inmunitaria con el fin de establecer el fin último de tal función (Vance, 2000), el problema de la individualidad, siempre irreductible a una ley general, parece eludir el discurso científico. Tal vez porque la identidad individual, en la especie humana, no se inscribe en una anatomía orgánica sino en el tejido particular de una historia perceptual y afectiva de la experiencia corporal. Al respecto, un biólogo como Francisco Varela ha podido afirmar que, en las condiciones humanas de organización vital, la identidad no es determinada a nivel molecular sino que en su significación (Varela, 1991).

En este sentido, la "escucha" del sufrimiento individual sería el complemento necesario a la objetividad que exije la observación médica - que privilegiando la eficacia de la intervención excluye toda subjetividad:

... la exigencia de reintroducir un valor ético en medecina es una consecuencia de la relacion inversa entre el "progreso" en el acercamiento a la materialidad biológica del cuerpo y la aproximación a la experiencia corporal... (Gori \& Del Volgo, 2005, p. 78)

El psicoanálisis, que nace con la exclusión que impone la medicina a la pantomima sintomática de la histeria, organiza su saber sobre un YO que es ante todo corporal. Pero Freud ha establecido las bases que permiten sostener que este cuerpo no corresponde solamente al de la fisiología, sino que es producido en una apropiación, siempre individual, de ésta. La "naturaleza cultural" de nuestra especie, efecto del apoyo y el apego que buscamos en el prójimo desde el nacimiento y del "apuntalamiento" del cuerpo de la cría humana que ésta 


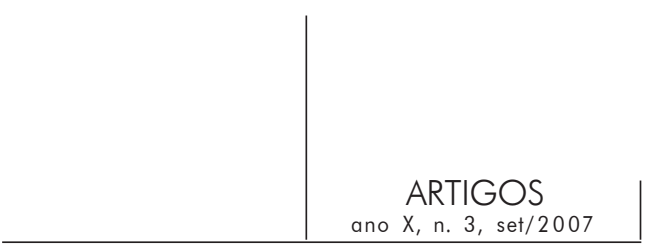

experiencia determina, redobla la fisiología de una organización desirante. Los órganos y funciones corporales se inscriben así en una lógica de sentido. A tal punto que el sufrimiento es capaz de generar una afección somática; el valor simbólico de la enfermedad afecta, a su vez, la vida afectiva.

Aunque estas afirmaciones son conocidas y aceptadas por la medicina, no hacen parte de la investigación ni de la intervención médicas. Lo que no es responsabilidad de la medicina: la compleja relación que establece el psicoanálisis al cuerpo lo ha llevado a una repugnancia respecto de su aspecto REAL. En consecuencia, incluso la construcción "psicosomática” establece una reducción positivista más cercana a la medicina que al psicoanálisis en afán fallido por evitar el dualismo que concierne necesariamente la manifestación somática - es el caso de H. Dunbar, F. Alexander, J. Nemiah, P. E. Sifneos, P. Marty - cuando no permanece en un misticismo incapaz de organizar un cuerpo teórico - $\mathrm{G}$. Groddeck, A. Garma. Sólo Winnicott parece respetar la inpensable dualidad del cuerpo humano sin por ello reducirlo a ninguno de sus componentes. Justamente, a través de los conceptos de self y de psicosoma.

El concepto de self encuentra su fundamento en el pensamiento de David Hume, para quien la identidad individual es el efecto de la progresión ininterrumpida de un pensamiento capaz de establecer una continuidad causal entre los diferentes instantes perceptuales del individuo. A partir de este antecedente, Heinz Hartmann introduce el concepto de self al psicoanálisis en la decada de los 50 para referirse al conjunto de las instancias susceptibles de devenir objeto de la pulsión narcísica. Melanie Klein lo utilizará a continuación para referirse al conjunto pulsional capaz de establecer de una diferencia entre el "mundo interior" y el Yo. Pero es con Donald Winnicott, que atribuye al self la capacidad de crear el sentimiento de realidad y de continuidad de la vida psíquica, "ligando" la experiencia perceptual al pensamiento, que el self encuentra su sentido definitivo en psicoanálisis. Esta idea de self no deja, sin embargo, de presentar dificultades conceptuales, presentándose sobre todo en su forma negativa, como falso self, aquel que sirve a proteger el "self autentico".

\section{Fenómenos auto-inmunes: ¿enfermedad o sufrimiento?}

El ideal totalitario de salud impuesto por la sofisticación de la medicina tecnocientífica, en su afán por medir, normalizar y homogenizar, excluye la singularidad propia a la clínica. La medicina ha pagado su cientificidad con un distanciamiento respecto de aquel cuerpo que sirve de sustrato a la experiencia vivida. Sin lamentar los avances de la técnica, “... esta nueva semiología (...) exige que otros se 


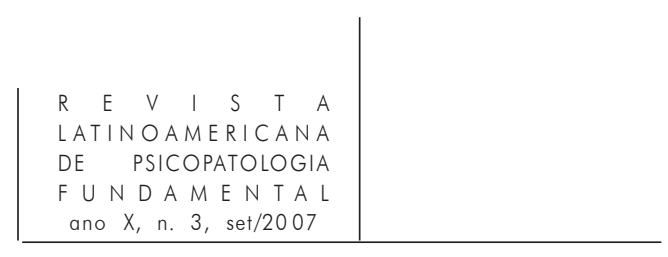

ocupen de la dimensión psicopatológica del sufrimiento psíquico producido por la enfermedad orgánica..." (Gori \& Del Volgo, 2005, p. 83). Además de la eficacia, un lugar para la subjetividad es necesario; para una clínica individual y ética. En efecto, en nuestra presencia ocasional en el hospital en tanto psicoanalistas constatamos la distancia establecida entre la dimensión orgánica de la enfermedad y el sufrimiento siempre asociado a ésta; la necesidad, en suma, de crear las posibilidades para un acercamiento psicoanalítico al cuerpo dentro del cuadro hospitalario.

La subjetividad crea su espacio, ciertamente, de manera espontánea a través de los juegos de saber y poder que se establecen entre médico y paciente. A pesar de ello, nos parece necesario crear las condiciones de apertura a la subjetividad, especialmente en casos extremos, cuando la presencia de la muerte llama al sujeto - al paciente, pero también al médico - a desaparecer detrás de un discurso docto, cargado de certeza o cuando el cuerpo sirve de inscripción, en acto, a un conflicto psíquico irrepresentable - incapaz de establecer la continuidad de sentido que caracteriza la vida si no es por el "triunfo de tanatos" (McDougall, 1996, p.154). En esta situación, sólo la presencia de un TU, otro que habla y presta su propia carne a la inscripción, hace posible la emergencia del sujeto en tanto YO que padece la enfermedad en una dimensión que excede la fisiología. “... la carne hace cuerpo por intermedio del interlocutor simbólico..." (Gori \& Del Volgo, 2005, p. 99).

Sylvia, vista en aislamiento de cuidados intermedios cuando toda intervención médica resultaba infructuosa, nos conduce ésta reflexión.

Sylvia, 42 años, casada y madre de dos hijos, comenzó a manifestar signos de una destrucción de la dermis y epidermis de origen auto-inmune pocas semanas después de la muerte de su padre. La enfermedad había evolucionado de manera brutal, llevándola a la pérdida de la piel de parte importante de la superficie corporal. Cuando la vi por primera vez, fue durante una visita del equipo de inmunología a la sección de aislamiento. Esta visita hacía parte de una discusión entre varios equipos de especialidad cuando la ausencia de resultados llevaba a la decisión de suspender los repetidos aseos quirúrgicos e intentos de injerto, así como los caros antibióticos prescritos. Sylvia, de mirada ausente, ocupaba una cámara de vidrio que la exponía a todas las miradas del piso. Se hablaba de ella durante la reunión de equipo sin que eso la interpelara o la hiciera reaccionar.

Al comenzar mis visitas cotidianas, no obtuve en un primer tiempo más que escasos monosílabos automáticos. Ella seguía ausente. Se estableció sin embargo poco a poco un dialogo entre ambos en torno a las visitas de su familia y las trazas que estos dejaban en su habitación. Comenzó así a relatar, casi sin darse cuenta, la novela de su vida, lo que la trajo paulatinamente de regreso a su cuerpo. El primer signo reconocible de esta habitación subjetiva fue el gesto de 


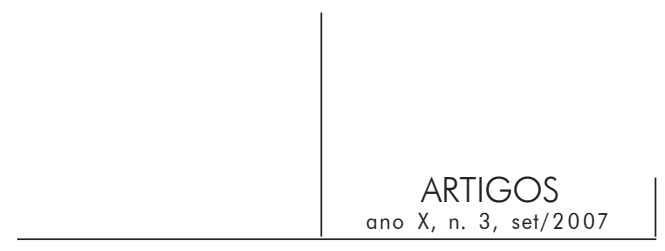

tomar la sábana que protegía negligentemente su cuerpo para cubrir el único resto de pezón que conservaba, al verme llegar desde el fondo del corredor una mañana. Cubriendo su feminidad ante mí, volvía a dar un valor simbólico a su cuerpo. Es en ese mismo período que Sylvie comienza a tomar una posición respecto de las intervenciones a las que era sometida regularmente, exigiendo la observación y discusión con los cirujanos antes de su traslado al pabellón quirúrgico. Pudo también hablar de su abandono de todo deseo y la pérdida de sentido en la que se había sumergido después de la muerte de su padre, así como de la culpabilidad con la que había vivido esta incapacidad de seguir viviendo, ante la angustia de su familia. Comenzó pronto a responder al tratamiento antibiótico y a los injertos comenzaron a "prender".

Este seguimiento de varios meses, reducido a algunas líneas con el propósito de esta comunicación, no podría dar cuenta del lugar del analista ante este cuerpo deshabitado, ni de los aspectos en juego en el abandono subjetivo, la relación de éste al desarrollo de la enfermedad ni de aquello que lograría dar un sentido, ligar, re-habitar un cuerpo en sufrimiento. Esta ilustración no ha pretendido más que subrayar la importancia de la presencia de otro sujeto ante este trastorno de la inmunidad - en la subjetivación de la enfermedad, de la medicina y del hospital. El psicoanálisis, mas allá de toda discusión respecto de los dispositivos y límites técnicos, tiene un lugar que ganar en este espacio. Pero si medicina y psicoanálisis comparten una pregunta respecto de un cuerpo común en sufrimiento, ¿qué lugar otorgar al psicoanálisis?

Aunque el surgimiento del psicoanálisis está estrechamente ligado a la erradicación de toda subjetividad que impone el modelo médico a fines del siglo XIX, éste abandona paulatinamente su lugar en los hospitales, privilegiando la neurosis, la transmisión y la institucionalidad. Si la re-obtención de un lugar para el sujeto dentro de la institución hospitalaria excede el movimiento personal de algunos psicoanalistas para ser reconocido como un problema que cuestiona los limites, la ética, y el lugar político - en tanto acepta las condiciones culturales de la polis - del psicoanálisis, es necesario que éste ultimo cree los discursos posibles para este intercambio. Este discurso, necesario para el diálogo con los otros saberes del cuerpo, debe exceder la dimensión puramente anatomo-fisiológica sin perder en ello el uno-a-uno que exige el espacio psicoanalítico. Estableciendo una complementariedad y no una rivalidad respecto del saber médico, no puede ser enigmático ni oscuro sino abrir la comunicación. Este discurso, que servirá a dar cuenta del trabajo analítico en su intercambio con la medicina, es igualmente importante para la transmisión del psicoanálisis. Se trata, entonces, de un lenguaje particular a las condiciones de transmisión del psicoanálisis y a las condiciones de inenteligibilidad del enigma dual del psiquesoma. 


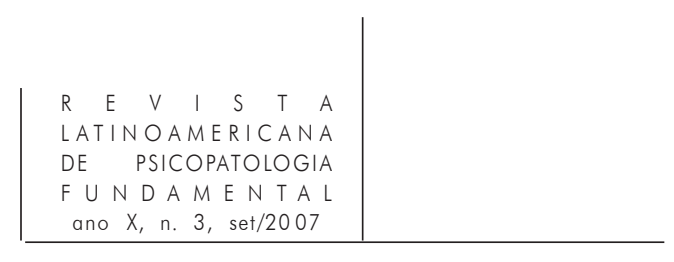

Este es el interés principal de esta mirada sobre la inmunología y el acercamiento médico al cuerpo individual. Aunque la identidad individual y el concepto de self, que constituye un recurso a la filosofía, parecen escapar al dominio puramente científico, la pregnancia de esta metáfora demustra la necesidad de un discurso respecto de este problema. En efecto, si para el clínico el self más que un paradigma representa una realidad capaz de determinar una perspectiva integrativa de la función inmunitaria en tanto defensa de "sí mismo" (Moulin, 2004, p. 629) a partir de la cual se establecen categorías diagnosticas y de intervención, es en este mismo trabajo "de terreno", y no en una construcción teórica, que sería posible abrir un espacio para el psicoanálisis.

Para comunicar su trabajo, el psicoanalista debe limitarse a los intercambios verbales de una o varias sesiones. Pierre Fédida nos recuerda que es, sin embargo, en la transferencia que se juega el acto analítico. Esta no se limita a la interacción conversacional sino que debe ser leída entre líneas (Fédida, 2000, p. 7), como si se tratase de una novela. Porque para apropiarse de la enfermedad es necesario inscribirla en la historia personal y la novela familiar que significan el cuerpo. Sólo estas condiciones particulares permiten establecer una continuidad entre la preocupación médica por la nosología y la prescripción, por una parte, y el sentido individual del sufrimiento y la enfermedad. Igualmente, respecto de la historia de la medicina, el concepto de self, más en tanto vocablo que como palabra-saber, articula medicina y psicoanálisis: en su indeterminación, self es tanto lo propio como el sí-mismo, el núcleo interior de individualidad, el doble por reflejo, la integridad orgánica, la continuidad de la existencia y el código genético de identidad. El vocablo, en cambio, más que significar hace del lenguaje un acto corporal, dando así a la palabra una dimensión de no-represión (Fédida, 2000, p. 14). Más allá de su definición, el vocablo self constituiría una superficie de extensión psíquica para la dimensión enigmática del cuerpo, evocando, por identificación, la sensación de necesidad de individuación y de integración tanto en el médico como en el paciente. El self es de aquellos conceptos que resisten a la transparencia de la descripción científica pero que sirven de pivote para el diálogo entre disciplinas y la integración de aquella parte esencial a la vida que no puede ser reducida a un análisis fisiológico - si no es posible establecer una base orgánica de identidad, es porque un cierto desacuerdo entre la ley general y el caso particular es necesario a la individualidad del viviente; los movimientos de vida, condicionados siempre por la movilidad y el azar, son irreductibles a una ley. Esta imposible predictibilidad, que determina el aspecto enigmático del cuerpo, justifica la atención al relato que un sujeto hace de su vida que propone este comentario. La medicina no puede, entonces, pasar por sobre el "lenguaje común" con el que el paciente habla de su enfermedad con el pretexto de la claridad diagnóstica, ya que sólo los significantes de la lengua materna, la lengua de 


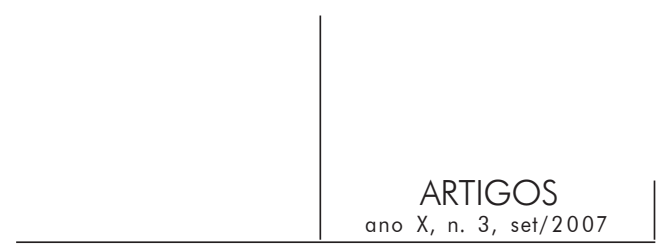

infancia, actualizan la problemática psíquica. Argumento que no pretende "... deconstruir este discurso para analizarlo en la transferencia como predicado de lo infantil..." (Gori \& Del Volgo, 2005, p.103) sino que supone el establecimiento de un efecto simbólico del síntoma, la enfermedad y los cuidados prodigados por el personal de salud en la enunciación de esta palabra "llena". El lenguaje médico es, en cambio, una lengua extranjera a la realidad psíquica.

\section{Conclusión}

La complejidad del sistema inmune, así como su distribución difusa en todo el organismo, determinan su carácter casi metafísico, sitúandolo en una interfase entre medicina y los otros saberes del cuerpo. El concepto de self, comodín que escurre entre discursos diferentes, ha servido de sostén a una ortopedia de integración del cuerpo fragmentado por las especialidades de la medicina moderna, sosteniendo la ilusión de individualidad de un cuerpo adaptado a la norma. Esta explicación, posible gracias a una cierta obscuridad conceptual, ha servido a la organización de la investigación, la nosología y las intervenciones médicas sobre el cuerpo sobre una base teórica que no se ampara de la solidez que exige la ciencia sino que constituye una apropiación fantasmática del cuerpo biológico; recreación del mito de unidad psiquesoma con el que la medicina se reapropia de enfermedades inclasificables o mal explicadas, hasta entonces bajo la indeterminación del "factor psicológico" (Moulin, 1991, p. 239).

A cien años del nacimiento de la inmunología, y cuando el artificio de aproximación que ha permitido el establecimiento de las condiciones individuación del cuerpo orgánico resulta insuficiente, una mirada a la adecuación y transparencia totales del que exije la medicina en su acercamiento a la disfunción, así como un análisis de la situación de la dimensión de sufrimiento de la enfermedad, resultan necesarias.

Ya sea que nos refiramos a la singularidad de la clínica, a la individualidad o incluso a la nosología, el lugar de lo subjetivo, que se resiste a toda inclusión en una ley general, se ha visto especialmente favorecido por la nebulosa conceptual que rodea a la inmunología. Esta misma inadecuación ha servido a un acercamiento posible la dimensión enigmática que representa a la experiencia corporal, el cuerpo habitado por un sujeto de lenguaje y cultura. El espacio necesario a los movimientos de vida, movimiento que liga, que se dirige al cuerpo del otro, que cruza en su recorrido el de otros sujetos, es un espacio de deseo

419




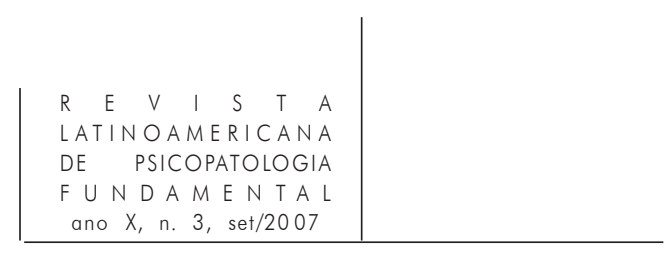

sólo posible en esta inadecuación entre la ley que lo describe y la actualidad de la contingencia. La vida, en los intersticios de toda descripción, es inasible. El goce del cuerpo es solo posible en esta zona de frontera, no man's land de descarga, intercambio, acto, rito.

Aunque el equivoco que rodea el self es impensable en ciencias, nos permite establecer las condiciones para hacer pensable lo humano, su irreductibilidad a una ley general y por la misma via, a un diálogo posible entre la medicina y el fundamento psicoanalítico de la individualidad de la clínica. Nos parece que el terreno clínico de los trastornos de la inmunidad, es su desafío al saber médico y en el sufrimiento del paciente ante lo irrepresentable de la enfermedad invisible e interior, permite estas condiciones de diálogo, que se sostienen en el presente texto del self.

\section{Referencias}

BuRnet, Frank McFarland. The Clonal Selection Theory of Acquired Immunity. Nashville: Vanderbilt University Press, 1959.

DAvID-Menard, Monique e HuMPHREYs, Derek. Self: immune matter or body enigma? In: L'évolution psychiatrique. Paris (in press), 2006.

FEDIDA, Pierre. Par où commence le corps humain. Paris: PUF, 2000.

Gori, Roland e Del Volgo, Marie Josée. La santé totalitaire: essai sur la médicalisation de l'existence. Paris: Denöel, 2005.

Matzinger, Poly. Tolerance, danger, and the extended family. Annual Review of Immunology, Palo Alto, CA: v. 12, april 1994.

McDougall, Joyce. Eros aux mille et un visages. Paris: Gallimard, 1996.

MouLin, Anne Marie. Le dernier langage de la médecine. Paris: PUF, 1991.

Moulin, Anne Marie. Immunité. In: Lecort, Dominique (sous la direction de) Dictionnaire de la pensée médicale. Paris: PUF, 2004. p. 629-30.

Tauber, Alfred. The immune self: Theory or Metaphor? Cambridge: Cambridge University Press, 1994.

VAnCE, Russell. Cutting Edge Commentary: A Copernican Revolution? Doubts About the Danger Theory. The Journal of Immunology, Bethesda, MD: v. 165, n. 4, aug. 2000.

VArela, Francisco. Organism: A meshwork of selfless selves. In: Tauber, Alfred (ed.)

Organism and the Origin of self. Dordrecht: Kluwer Assoc., 1991. p. 79-107. 
A cientificidade da medicina moderna, ao se distanciar da particularidade do caso clínico, encontra no conceito de self uma metáfora da individualidade. Partindo das dificuldades próprias a esse conceito, o presente artigo analisa a individualidade biológica e sua relação com a psicanálise, com o intuito de estabelecer um diálogo a respeito dos distúrbios somáticos. A abertura de um espaço de subjetividade, especificamente no desafio ao saber médico dos transtornos da imunidade, introduz uma dimensão humana ao sofrimento orgânico, permitindo estabelecer tal diálogo, através do self.

Palavras-chave: Individualidade biológica, psicanálise, transtorno somático, psicopatologia fundamental

Dans une scientificité qui l'éloigne de la particularité du cas clinique, la médecine moderne retrouve une métaphore d'individualité dans le concept de self. L'individualité biologique et son rapport à la psychanalyse sont ici analysés à partir des difficultés liées à ce concept dans le but d'établir un dialogue sur la question des troubles somatiques. L'ouverture d'un espace de subjectivité, possible notamment par les questions que le savoir médical se pose face aux troubles de l'immunité, introduit une dimension humaine à la souffrance organique, rendant possible ce dialogue à travers le concept de self.

Mots clés: Individualité biologique, psychanalyse,troubles somatiques, psychopathologie fondamentaux

The scientific character of modern medicine has been put at a distance from the particularity of clinical cases, and the concept of "self" has thus become a metaphor of individuality. Based on the difficulties introduced by this concept, the present article analyses biological individuality and its relations to psychoanalysis, in order to establish a discussion on somatic disorders. The challenge that immunity disorders present to medical knowledge bring up the human dimension in organic suffering, consequently permitting a place for subjectivity. This dialogue becomes possible through the "self."

Key words: Biological individuality, psychoanalysis, somatic disorders, fundamental psychopathology 